OPEN ACCESS

Edited by:

Cesare Indiveri,

University of Calabria, Italy

Reviewed by:

Michele Visentin,

University Hospital Zurich, Switzerland

Annamaria Tonazzi,

National Research Council (CNR), Italy

*Correspondence:

Giuliano Ciarimboli

gciari@uni-muenster.de

Specialty section:

This article was submitted to

Cellular Biochemistry,

a section of the journal

Frontiers in Cell and Developmental

Biology

Received: 31 March 2021 Accepted: 30 April 2021

Published: 28 May 2021

Citation:

Schorn S, Dicke A-K, Neugebauer U, Schröter R,

Friedrich $M$, Reuter $S$ and Ciarimboli G (2021) Expression and Function of Organic Cation

Transporter 2 in Pancreas. Front. Cell Dev. Biol. 9:688885. doi: 10.3389/fcell.2021.688885

\section{Expression and Function of Organic Cation Transporter 2 in Pancreas}

\author{
Sandra Schorn, Ann-Kristin Dicke, Ute Neugebauer, Rita Schröter, Maren Friedrich, \\ Stefan Reuter and Giuliano Ciarimboli*
}

Experimental Nephrology, Medicine Clinic D, University Hospital Münster, Münster, Germany

Organic cation transporters (OCT) play an important role in mediating cellular uptake of several pharmaceuticals, such as the antidiabetic drug metformin and the platinumderived chemotherapeutics. Since these drugs can also affect the pancreas, here it was investigated whether these transporters are expressed in this organ. An interaction between OCT2 and the glucose transporter 2 (GLUT2), which is expressed with important functional consequences in the kidneys and in the pancreas, has already been demonstrated elsewhere. Therefore, here it was further investigated whether the two proteins have a functional relationship. It was demonstrated that OCT2 is expressed in pancreas, probably in $\beta$ cells of Langerhans islets, together with GLUT2. However, a co-localization was only evident in a cell-line model of rat pancreatic $\beta$ cells under incubation with high glucose concentration. High glucose stimulated OCT2 expression and activity. On the other side, studies conducted in human embryonic kidney cells stably expressing OCT2, showed that overexpression of GLUT2 decreased OCT2 activity. Unfortunately, pull-down experiments aimed to confirm a physical OCT2/GLUT2 interaction were not successful. Renal glucose excretion was reduced in mice with genetic deletion of OCT2. Nonetheless, in these mice no regulation of known kidney glucose transporters was measured. Therefore, it may be speculated that OCT2 may influence cellular trafficking of GLUT2, without changing its amount. OCT2 may play a role in drug uptake of the pancreas, and its activity may be regulated by glucose and GLUT2. Vice versa, GLUT2 activity may be regulated through an interaction with OCT2.

Keywords: organic cation transporters, glucose transporter, glucose, pancreas, metformin

\section{INTRODUCTION}

Metformin is a broadly used antidiabetic drug with glucose-lowering effects. It seems that metformin main mechanisms of action derive from inhibition of hepatic gluconeogenesis and improved glucose uptake in skeletal muscles (Pernicova and Korbonits, 2014). Metformin has also an effect on pancreas function restoring insulin secretion and protecting pancreatic $\beta$ cells from lipotoxicity or glucotoxicity (Yang et al., 2017). Cellular metformin passage through the plasma membrane is mainly mediated by organic cation transporters (OCT) and multidrug and toxin extrusion proteins (MATEs) (Zhou et al., 2016). Polymorphisms of the genes encoding for these transporters may change cellular transport of metformin, despite that the question whether these mutations have a clinical significance is still debated (Zazuli et al., 2020). Interestingly, a mutation in the Solute Carrier 2A2 (SLC2A2) gene encoding for the glucose transporter 2 
(GLUT2) has been demonstrated to have a clear clinical impact on metformin efficacy especially in obese patients (Zhou et al., 2016). GLUT2 is expressed in the central nervous system, in the liver, intestine, kidneys, and pancreatic islet $\beta$ cells, where, at least in rodents, it is required for glucose-stimulated insulin secretion (Thorens, 2015). In enterocytes, hepatocytes, and in epithelial cells from the kidney proximal convoluted tubule epithelial cells, GLUT2 is expressed mainly in the basolateral membrane domain. However, in enterocytes after a sugar meal (Leturque et al., 2009) and in renal proximal tubules in dependence from increasing glucose concentrations (Marks et al., 2003), GLUT2 can translocate to the apical membrane. Using a mating-based split-ubiquitin-system (mbSUS), a protein-protein interaction between GLUT2 and the human OCT2 (hOCT2) was observed (Snieder et al., 2019). Because hOCT2 is a transporter for metformin (Song et al., 2008; Konig et al., 2011) and metformin also acts on pancreas (Yang et al., 2017), in this brief research report we investigated, whether OCT2 is expressed in pancreatic tissue and whether GLUT2 and glucose can regulate its activity.

\section{MATERIALS AND METHODS}

\section{Cells}

As a model for pancreatic $\beta$ cells the INS-1 832-13 cells (Duke Molecular Physiology Institute, Duke University, Durham, NC, United States) (Hohmeier et al., 2000), which are cells isolated from X-ray induced rat-insulinoma and can secrete insulin, were used. The cells were cultured in Roswell Park Memorial Institute medium [RPMI 1640, Sigma-Aldrich, Taufkirchen, Germany (Hohmeier et al., 2000)] supplemented with $10 \%$ fetal calf serum (FCS), 1\% penicillin/streptomycin, $10 \mathrm{mM}$ HEPES, $1 \mathrm{mM}$ glutamine, $2 \mathrm{mM} \mathrm{Na}^{+}$-pyruvate and $0.05 \mathrm{mM}$ 2-mercaptoethanol. In some experiments, glucose concentration was increased from 9.8 to $14.8 \mathrm{mM}$. Osmolality in control cells was adjusted by mannose addition.

The effect of transfection with human GLUT2 (hGLUT2, a gift from RESOLUTE Consortium \& Giulio Superti-Furga, Addgene plasmid \#132126 ${ }^{1}$ ) cloned into a pcDNA DEST47 vector) was studied in human embryonic cells (HEK) stably over-expressing the human OCT2 [hOCT2, for a detailed description of these cells see Biermann et al. (2006)]. Transfection was performed using the calcium phosphate transfection method. $72 \mathrm{~h}$ after transfection with GLUT2 or the empty vector (EV), the cells were used for $\mathrm{ASP}^{+}$uptake measurements and western blot analysis, as described below.

Immunoprecipitation experiments with lysates from HEK cells transfected with hOCT2 tagged with green fluorescent protein (GFP) at the carboxy-terminus (hOCT2GFP) (as described in Brast et al., 2012) and GLUT2 were performed using GFP trap agarose beads (ChromoTek, PlaneggMartinsried, Germany). Briefly, cells were lysed with Laemmli buffer [10\% SDS, 20\% (v/v) Glycerol, $125 \mathrm{mM}$ Tris-HCl (pH 6.8)] containing $200 \mathrm{mM}$ dithiothreitol and $0.004 \%$ bromophenol blue. Lysates were incubated $2.5 \mathrm{~h}$ at $4^{\circ} \mathrm{C}$ under rotation with GFP

${ }^{1}$ http://n2t.net/addgene:132126 trap agarose beads. The immunocomplexes bound to the beads were recovered by centrifugation, washed five times, resuspended in Laemmli buffer, denaturated at $95^{\circ} \mathrm{C}$ for $5 \mathrm{~min}$, and loaded onto 4-20\% SDS-polyacrylamide gels (Mini-Protean TGX gels, Bio-Rad, Hercules, CA, United States). For western blot analysis the proteins on the gel were transferred to a nitrocellulose membrane incubated with blocking reagent [3\% milk powder and $1 \%$ bovine serum albumin (BSA) in Tris-buffered saline with Tween20 (TBST)]. After incubation with the primary antibody (1:500 rabbit-polyclonal antibody to hOCT2, Sigma-Aldrich, Munich, Germany, or 1:1000 rabbit-polyclonal antibody to hGLUT2 Proteintech, St. Leon-Rot, Germany, or 1:1000 rabbit antibody against $\alpha$-actinin, Cell Signaling Technology, Frankfurt, Germany), membranes were incubated with anti-rabbit HRPconjugate (1:10,000 Thermo Fisher Scientific, Waltham, MA, United States), then covered with Lumi-Light or Lumi-Light Plus (Roche, Mannheim, Germany) before exposure (c600 azure biosystem imager, Azure Biosystems, Dublin, CA, United States).

\section{Fluorescence Measurements}

The fluorescent organic cation $\mathrm{ASP}^{+}$was used as a tracer of OCTmediated transport, as already described in detail (Wilde et al., 2009; Wittwer et al., 2013). Measurements were performed using a microplate fluorescence reader with excitation at $465 \mathrm{~nm}$ and emission at $590 \mathrm{~nm}$ (Infinite F200, Tecan, Switzerland) (Wilde et al., 2009). $\mathrm{ASP}^{+}$-concentration was $1 \mu \mathrm{M}$ in experiments with hOCT2-expressing HEK cells and $5 \mu \mathrm{M}$ for experiments with INS-1 cells.

\section{PCR Analysis}

For PCR analysis, total RNAs were isolated using the Qiagen RNeasy Minikit (Qiagen, Gilden, Germany) and reverse transcription was performed using the Superscript II system (Invitrogen, Carlsbad, CA, United States), both according to the manufacturer's recommendations. Standard PCR was performed using specific primer pairs as listed in Supplementary Table 1. The PCR products were separated using agarose gel electrophoresis. The bands were sequenced for confirmation of amplification product identity.

\section{Animals}

Male OCT2 ${ }^{-/-}$mice (Prof. Schinkel, The Netherlands Cancer Institute, Amsterdam, Netherlands) on Bl6 background, and male Bl6 wild-type (WT) mice approximately 9 weeks old and weighing 25-30 g were used. Compared with WT animals, OCT2 $2^{-/-}$mice display no obvious phenotypic abnormalities (Jonker et al., 2003). Experiments were approved by a governmental-committee on animal welfare (84-02.04.2014.A454) and were performed in accordance with national animal protection guidelines. Mice were placed in metabolic cages and $24 \mathrm{~h}$ urines were collected for the determinations of glucose excretion. Glucose was measured by the hexokinase method with a Roche Hitachi Modular automatic analyzer (Gluco-quant Glucose, Roche, Mannheim, Germany). In some experiments, pancreas tissues from mice and rats were collected. For immunofluorescence analysis, pancreas was removed and fixed with $4 \%$ neutral buffered formalin. Expression 
of OCT2 and GLUT2 in mouse pancreas was investigated using specific antibodies raised against mouse OCT2 (1:300, a generous gift from Prof. Koepsell) and against GLUT2 (1:50, Glut2 C19 SC-7580, Santa Cruz Biotechnology, Dallas, TX, United States). Five micrometer thick cryosections were prepared from mouse pancreas, which have been fixed in melting 2-methylbutane. Cryosections were incubated with $0.2 \%$ Triton in phosphatebuffered saline (PBS) for $5 \mathrm{~min}$ at room temperature, three times washed with PBS and then incubated $1 \mathrm{~h}$ with a 1:1 mix of $10 \%$ BSA and 10\% normal human serum (NHS) in PBS. After this, sections were incubated overnight at $4^{\circ} \mathrm{C}$ with primary antibodies. After washing in PBS, the sections were incubated for $45 \mathrm{~min}$ at room temperature in the dark with PBS-diluted secondary antibodies (donkey Alexa Fluor 488 anti- goat -Ig, and Alexa Fluor 647 anti- rabbit -Ig Invitrogen, 1:1,000). Sections were rinsed with PBS, coverslipped with Fluoromount aqueous mounting medium (Sigma-Aldrich, München, Germany) and evaluated by epifluorescence microscopy (Observer Z1 with Apotome, Zeiss). Negative control slides were included without addition of primary antibody.

\section{REAGENTS}

4-(4-dimethylaminostyril)-N-methylpyridinium $\left(\mathrm{ASP}^{+}\right)$was purchased from Fischer Scientific. All other reagents were of the highest purity and obtained from Sigma-Aldrich (Sigma-Aldrich, Merck Chemicals, Darmstadt, Germany).

\section{Statistical Analysis}

Experimental data are presented as means \pm SEM, with $n$ referring to the number of totally measured replicates obtained in at least three independent experiments. Significant differences were calculated using unpaired Student's $t$-test or ANOVA with Tukey's post-test for multiple comparisons. A $p$-value $<0.05$ was considered statistically significant. Analyses were performed using GraphPad Prism, Version 5.3 (GraphPad Software, San Diego, CA, United States).

\section{RESULTS}

Expression of GLUT2 and OCT2 was studied in pancreas tissues from mice, rats and in the INS-1 cells (Figure 1). Immunofluorescence analysis of protein expression in mouse pancreas shows that islets express GLUT2 in the plasma membrane, while OCT2 is visible only in some cells in intracellular compartments (Figure 1A). No co-localization of the two proteins was evident. Mouse pancreas expresses mRNA for GLUT1, GLUT2, and OCT2 (Figure 1B). For OCT2 more bands were detected. The thicker lower one corresponds to mOCT2, as confirmed by sequencing (the identity of all PCR-products was confirmed by sequencing). No specific band for OCT1 and OCT3 was detected. In rat pancreas (Figure 1C) an expression of OCT1- and OCT2-, but not of OCT3-mRNA was evident only upon reamplification of the PCR-products, which produced a double band for

\section{Mouse pancreas}

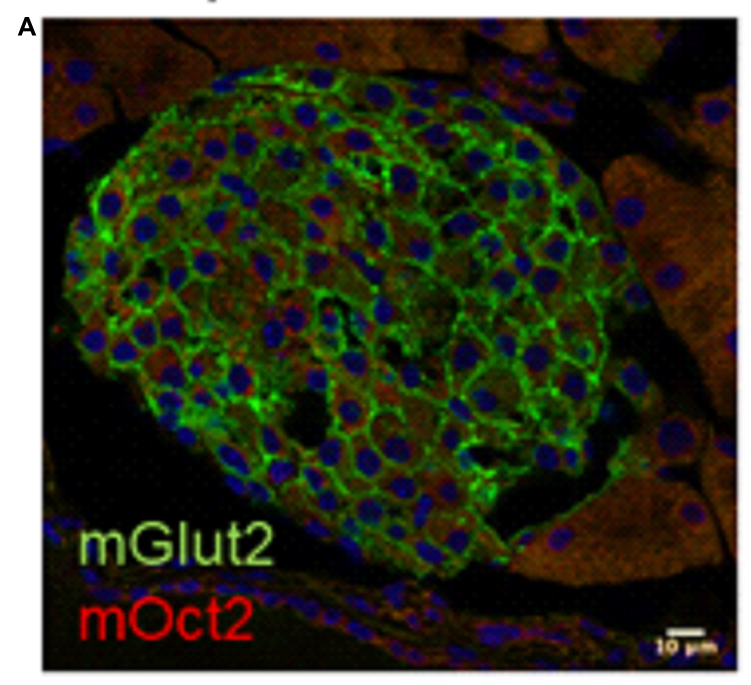

B

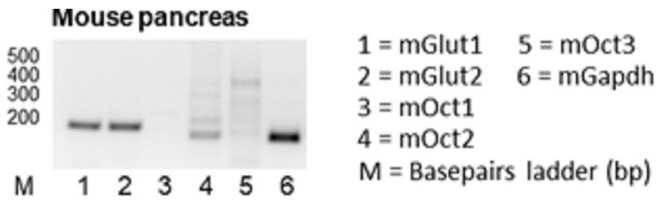

C

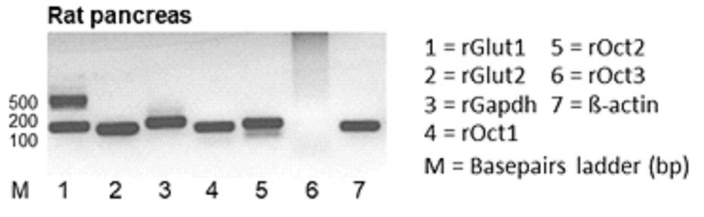

D

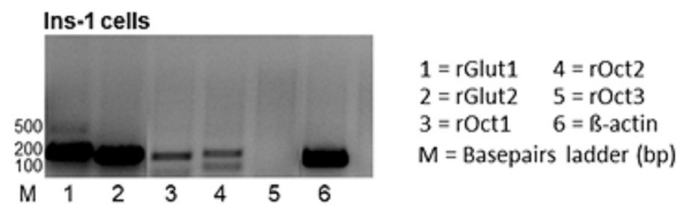

FIGURE 1 | This figure shows the expression of GLUT and OCT in the pancreas. In panel (A) a representative immunofluorescence analysis of GLUT2 (green) and OCT2 (red) expression in a mouse pancreatic islet is shown. GLUT2 is evident in the plasma membrane of islet cells. OCT2 seems to be expressed in intracellular compartments of some islet cells. No GLUT2/OCT2 co-localization is observed. Panel (B) shows that mouse pancreas expresses mRNA of GLUT1 and 2 and of OCT2, but not of OCT1 and 3. For OCT2 several bands are visible, but sequencing confirmed the identity of the lower band with OCT2. In rat pancreas (C) OCT expression became visible only after reamplification. However, this reamplification caused the appearance of an unspecific band for GLUT1. Sequencing confirmed the lower band to be specific for GLUT1. The INS-1 cells show the same transporter expression pattern as the rat pancreas (D).

GLUT1. This suggests that OCT1- and 2-mRNA expression is relatively low. For GLUT1, only the lower band is specific. GLUT2-mRNA is also robustly expressed in rat pancreas. Since the INS1-cells show a similar transporter expression pattern 

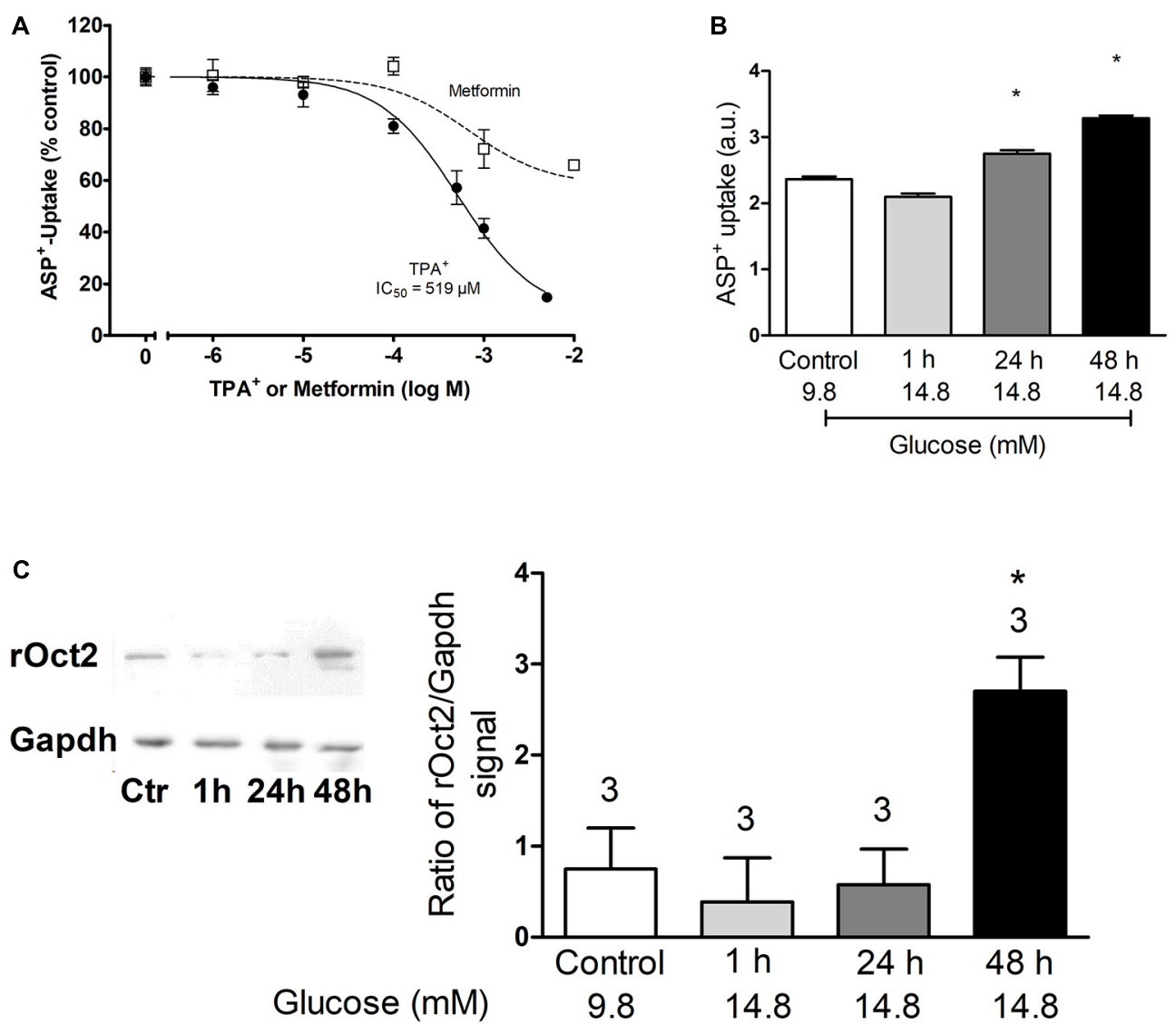

D rOct2

Glut2

\section{merge}

control
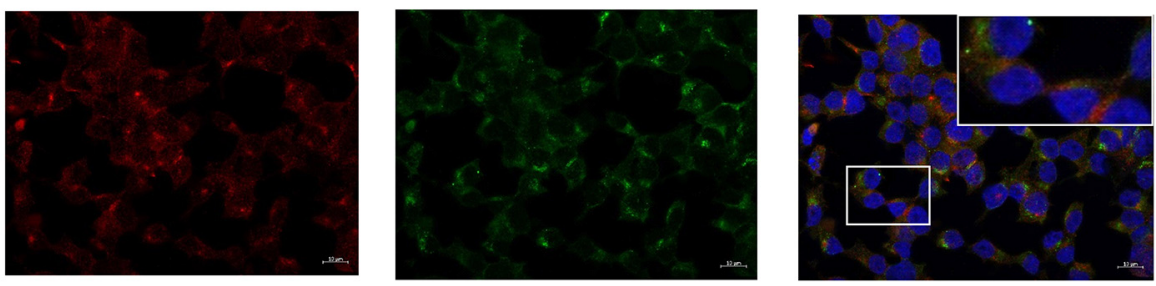

$48 \mathrm{~h}$

$14.8 \mathrm{mM}$ glucose
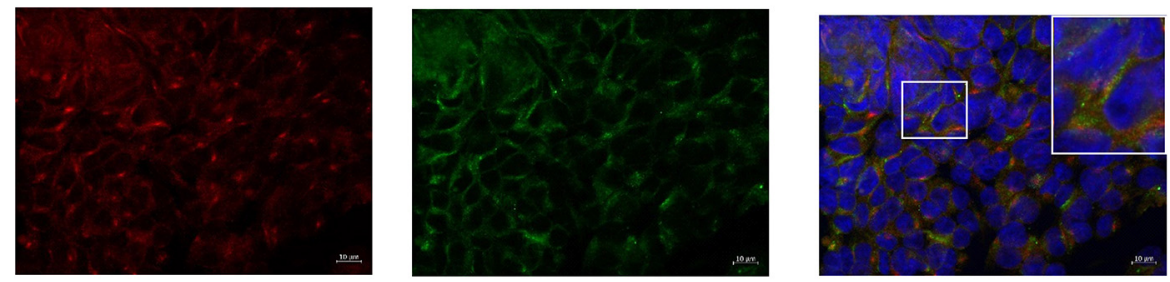

FIGURE 2 | This figure shows the ASP ${ }^{+}$transport characteristics of INS-1 cells and the analysis of OCT2 and GLUT2 expression in dependence from glucose concentration. Panel (A) shows that TPA ${ }^{+}$inhibits concentration-dependently the $\mathrm{ASP}^{+}$uptake with an $\mathrm{IC}_{50}$ of $519 \mu \mathrm{M}$. Inhibition of $\mathrm{ASP}^{+}$uptake by metformin is also shown (at least six replicates for concentration measured in three independent experiments). Panel (B) shows the effect of incubation with 14.8 mM glucose for 1, 24, or $48 \mathrm{~h}$ on the $\mathrm{ASP}^{+}$uptake in arbitrary units (a.u.) of INS-1 cells. Control experiments were performed with a glucose concentration of 9.8 mM. Osmolarity was adjusted in control experiments by adding mannose. High glucose significantly stimulated ASP ${ }^{+}$uptake after incubation for 24 and $48 \mathrm{~h}$ (ANOVA with Tukey's post-test, nine replicates measured in four independent experiments). Panel (C) shows the evaluation of western blot analysis of OCT2 expression under incubation with high glucose. Only after $48 \mathrm{~h}$ incubation with $14.8 \mathrm{mM}$ glucose a significant increase of OCT2 expression was observed $\left({ }^{*}\right.$, ANOVA with Tukey's post-test, three independent experiments). The insert on the left shows an example of the western blot analysis. Panel (D) shows the immunofluorescence analysis of OCT2 (red) and GLUT2 (green) expression in INS-1 cells after $48 \mathrm{~h}$ incubation with normal $(9.8 \mathrm{mM}$ ) or high (14.8 mM) glucose. The labeling of nuclei with

$4^{\prime}, 6$-diamidino-2-phenylindole (DAPI, blue) is also shown. In the upper right corners of the overlay picture a magnification of the field indicated by a box is shown. 

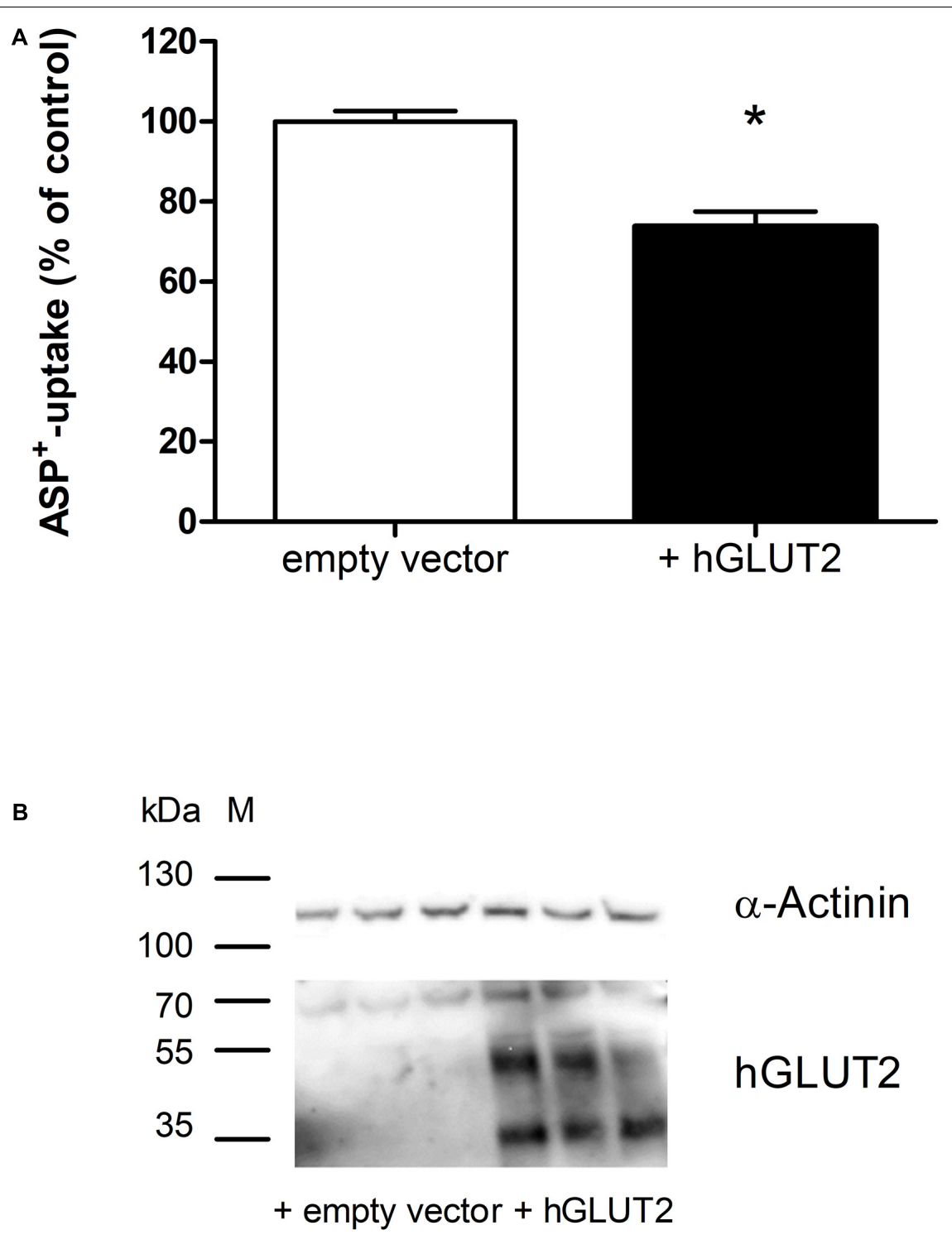

FIGURE 3 | Transfection of HEK cells stably expressing hOCT2 with GLUT2: effects on the ASP ${ }^{+}$uptake. Panel (A) shows the uptake of $1 \mu$ M ASP + in HEK cells

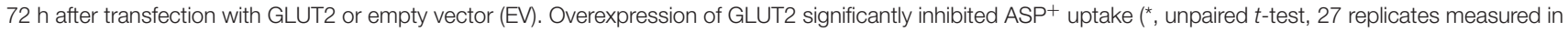
three independent experiments). Panel (B) shows the western blot analysis of GLUT2 $72 \mathrm{~h}$ after transfection. $\alpha$-Actinin was used as loading marker. This antibody against hGLUT2 is known to detect mature glycosylated hGLUT2 at 60-70 kDa, non-glycosylated hGLUT2 at 38-45 kDa, and a hGLUT2 fragment at around 35 kDa (https://www.ptglab.com/products/SLC2A2-Antibody-20436-1-AP.htm). All these bands are visible in this western blot analysis, especially in HEK cells transfected with hGLUT2, confirming that transfection with hGLUT2 was successful. Bands corresponding to GLUT2 were observed mainly in transfected cells.

(Figure 1D, also here a reamplification was necessary to detect OCT1- and OCT2-mRNA, the thicker band was specific for the transporters as revealed by sequencing), it was investigated, whether these cells show any $\mathrm{ASP}^{+}$-transport. INS-1 cells are able to take up $\mathrm{ASP}^{+}$(Figure 2A): this uptake was inhibited concentration-dependently by tetrapentylammonium $\left(\mathrm{TPA}^{+}\right)$, a known OCT-inhibitor (Zhang et al., 1999; Wilde et al., 2009), with an apparent affinity $\left(\mathrm{IC}_{50}\right)$ of $519 \mu \mathrm{M}$. Metformin, which is well known to interact with OCT, showed a lower but still significant inhibitory capacity than $\mathrm{TPA}^{+}$. Increasing glucose concentration from 9.8 to $14.8 \mathrm{mM}$ resulted in an increased uptake of $\mathrm{ASP}^{+}$after 24 and $48 \mathrm{~h}$ incubation (Figure 2B). Osmolality was equilibrated by mannose addition. Western blot analysis of the cells showed that rat OCT2 protein expression significantly increased after $48 \mathrm{~h}$ incubation with high glucose (Figure 2C). Immunofluorescence analysis of INS-1 cells after $48 \mathrm{~h}$ incubation with $14.8 \mathrm{mM}$ glucose suggests an increased cell proliferation (as already shown in Olson et al., 1998), and an increased expression of OCT2 and GLUT2, with augmented distribution to the plasma membrane (Figure 2D). In some cells, a partial co-localization of OCT2 and GLUT2 can be observed after $48 \mathrm{~h}$ incubation with $14.8 \mathrm{mM}$ glucose (Figure 2D, merge). 
Transfection of hGLUT2 in HEK cells overexpressing hOCT2 caused a significant inhibition $(-26 \pm 4 \%)$ of hOCT2 function measured as $\mathrm{ASP}^{+}$-uptake (Figure 3A). The transfection with hGLUT2 resulted in an evident GLUT2 protein expression (Figure 3B). The expression levels of hOCT2 were not changed (not shown). However, GLUT2 was not detectable in GFP-trap pull-down experiments (not shown).

The in vitro experiments suggest that high glucose and also GLUT2 protein expression can change OCT activity and cellular distribution. Since high amounts of GLUT2 and OCT2 are expressed in the basolateral plasma membrane domain of renal proximal tubules (Koepsell et al., 2007; Thorens, 2015), we tried to investigate whether OCT2 influences glucose kidney handling by comparing renal glucose excretion in WT- and OCT2 $2^{-/-}$mice (Figure 4). A decreased glucose urinary secretion was measured in OCT2 $2^{-/}$-compared to WT mice (Figure 4).

\section{DISCUSSION}

In this brief research report, it has been demonstrated that OCT 1 and 2 are expressed in the pancreas. Previously, expression of OCT1 was demonstrate in human pancreatic stellate cells (Wu et al., 2018). Focusing on OCT2, it seems to be expressed in some cells of the Langerhans islets. From experiments with the INS-1 cells, which are able to release insulin and are a model for $\beta$ cells, it can be speculated that OCT2 is expressed in the $\beta$-cells. Interestingly, a partial co-localization with GLUT2 was only detected under incubation with high glucose concentration, suggesting that probably in this cell system, a protein conformational change may be induced by high-glucose, which then facilitates OCT2-GLUT2 interaction. Increased OCT2 expression and function under incubation with high-glucose may facilitate metformin action under diabetic conditions. INS-1 cells can transport $\mathrm{ASP}^{+}$and this transport can be inhibited by $\mathrm{TPA}^{+}$with an apparent affinity, which is much

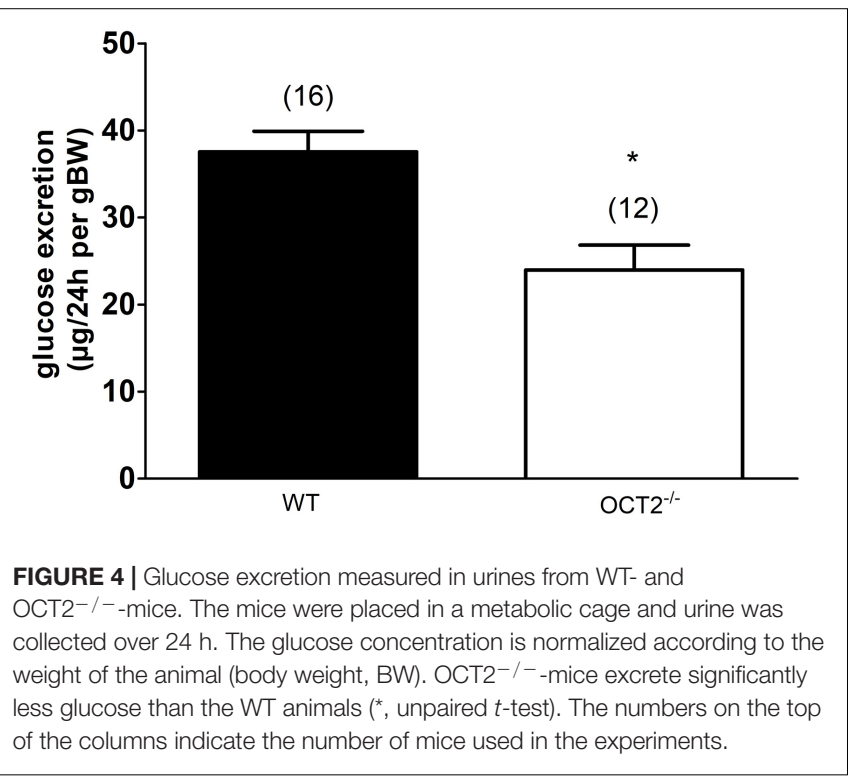

lower than that observed using rat OCT1 (rOCT1) (Ciarimboli et al., 2005) or rOCT2 (Wilde et al., 2009) in expression systems. This suggests that the apparent affinity can be changed by specific cellular regulation pathways (Ciarimboli et al., 2004). In INS1 cells, a low but significant inhibition of $\mathrm{ASP}^{+}$uptake by metformin was measured. This inhibition is lower than that measured using $\mathrm{ASP}^{+}$with cloned OCT (see Frenzel et al., 2019), again suggesting an influence of cell specific factors in determining transport characteristics.

Transfection of GLUT2 decreases the transport activity of hOCT2 overexpressed in HEK cells, suggesting that the hOCT2GLUT2 interaction detected using the mbSUS (Snieder et al., 2019) may have functional consequences. However, in pull-down experiments using GFP-trap and HEK cells transfected with hOCT2-GFP [the GFP tag is localized at the carboxy-terminus of the transporter and does not change its function, as already demonstrated in Brast et al. (2012)] it was not possible to confirm a direct hOCT2-GLUT2 interaction. It can be speculated, that the interaction domain is close to the carboxy-terminus, where hOT2 bears the GFP-tag. The tag may change the conformation of this part of the protein, hindering the interaction with GLUT2.

Interestingly, genetic deletion of OCT2 in mice caused a significant lower excretion of glucose compared to WT animals. In a previous work, performing a proteomic analysis of kidneys from WT- and OCT $1 / 2^{-/-}$mice no different expression of proteins involved in glucose resorption [sodium glucose transporter (SGLT)1 and 2, GLUT1 and 2] was detected (Hucke et al., 2019), suggesting that the difference in glucose excretion is not due to changes in global protein amount. Perhaps the OCT2/GLUT2 interaction changes the transport characteristics of these proteins or modify their trafficking from/to the plasma membrane. For example, in the absence of OCT2, GLUT2 may increase its transport activity or/and may traffic to the apical plasma membrane, in both ways increasing renal glucose reabsorption. Unfortunately, we were not able to analyze these aspects in the kidneys.

Platinum derivatives such as the chemotherapeutic drugs cisplatin and oxaliplatin, are also substrates of OCT (Sprowl et al., 2013). Anticancer treatment with these drugs is well known to cause severe side-effects such as nephrotoxicity and peripheral neurotoxicity, which are probably mediated by OCT-mediated uptake (Hucke et al., 2019; Sprowl et al., 2013). There is also some evidence that cisplatin and oxaliplatin can cause pancreatic toxicity (Komdeur et al., 2007; Yadav, 2019). Therefore, it may be speculated that OCT2 may play a role also in this type of unwanted side-effect of platinum-based chemotherapy.

Organic cation transporters have a large binding pocket, with specific interaction domains for different substrates. Therefore, they are polyspecific, meaning that they can accept many different substances as substrate (Koepsell et al., 2007). For this reason, OCT can mediate the cellular uptake of many different drugs and can be involved in cellular drug toxicity. While the OCT-mediated drug toxicity in organs like the kidneys and the liver is well known (Motohashi and Inui, 2016), pancreatic drug toxicity has not yet been associated with OCT expression. Many medications, which are associated with pancreas toxicity (Jones et al., 2015) are substrates 
of OCT, like prazosin, procainamide, ranitidine, and cimetidine (Koepsell, 2020).

\section{CONCLUSION}

In conclusion, here we demonstrated that OCT2 is expressed in the pancreas and that high-glucose stimulates its expression and co-localization with GLUT2. A possible functional role of this interaction is plausible, but we were not able to define the interaction mechanisms. Further studies are necessary to elucidate the characteristics of this interaction and whether it may have some functional implication.

\section{DATA AVAILABILITY STATEMENT}

The raw data supporting the conclusions of this article will be made available by the authors, without undue reservation.

\section{ETHICS STATEMENT}

The animal study was reviewed and approved by Landesamt für Natur, Umwelt und Verbraucherschutz Nordrhein-Westfalen.

\section{REFERENCES}

Biermann, J., Lang, D., Gorboulev, V., Koepsell, H., Sindic, A., Schröter, R., et al. (2006). Characterization of regulatory mechanisms and states of human organic cation transporter 2. Am. J. Physiol. Cell Physiol. 290, C1521-C1531.

Brast, S., Grabner, A., Sucic, S., Sitte, H. H., Hermann, E., Pavenstadt, H., et al. (2012). The cysteines of the extracellular loop are crucial for trafficking of human organic cation transporter 2 to the plasma membrane and are involved in oligomerization. FASEB J. 26, 976-986. doi: 10.1096/fj.11-180679

Ciarimboli, G., Koepsell, H., Iordanova, M., Gorboulev, V., Dürner, B., Lang, D., et al. (2005). Individual PKC-phosphorylation sites in organic cation transporter 1 determine substrate selectivity and transport regulation. J. Am. Soc. Nephrol. 16, 1562-1570. doi: 10.1681/asn.2004040256

Ciarimboli, G., Struwe, K., Arndt, P., Gorboulev, V., Koepsell, H., Schlatter, E., et al. (2004). Regulation of the human organic cation transporter hOCT1. J. Cell Physiol. 201, 420-428. doi: 10.1002/jcp.20081

Frenzel, D., Koppen, C., Bauer, O. B., Karst, U., Schroter, R., Tzvetkov, M. V., et al. (2019). Effects of Single Nucleotide Polymorphism Ala270Ser (rs316019) on the Function and Regulation of hOCT2. Biomolecules 9:578. doi: 10.3390/ biom 9100578

Hohmeier, H. E., Mulder, H., Chen, G., Henkel-Rieger, R., Prentki, M., and Newgard, C. B. (2000). Isolation of INS-1-derived cell lines with robust ATPsensitive $\mathrm{K}+$ channel-dependent and -independent glucose-stimulated insulin secretion. Diabetes 49, 424-430. doi: 10.2337/diabetes.49.3.424

Hucke, A., Rinschen, M. M., Bauer, O. B., Sperling, M., Karst, U., Koppen, C., et al. (2019). An integrative approach to cisplatin chronic toxicities in mice reveals importance of organic cation-transporter-dependent protein networks for renoprotection. Arch. Toxicol. 93, 2835-2848. doi: 10.1007/s00204-01902557-9

Jones, M. R., Hall, O. M., Kaye, A. M., and Kaye, A. D. (2015). Drug-induced acute pancreatitis: a review. Ochsner. J. 15, 45-51.

Jonker, J. W., Wagenaar, E., Van Eijl, S., and Schinkel, A. H. (2003). Deficiency in the organic cation transporters 1 and 2 (Oct1/Oct2 [Slc22a1/Slc22a2]) in mice abolishes renal secretion of organic cations. Mol. Cell Biol. 23, 7902-7908. doi: $10.1128 / \mathrm{mcb} .23 .21 .7902-7908.2003$

\section{AUTHOR CONTRIBUTIONS}

SS, A-KD, UN, RS, MF, SR, and GC performed and evaluated the experiments. GC planned the study and wrote the manuscript. All authors contributed to the article and approved the submitted version.

\section{FUNDING}

This study was supported by the Deutsche Forschungsgemeinschaft (CI 107/11-1 to GC).

\section{ACKNOWLEDGMENTS}

The help of Christopher Newgard (Duke Molecular Physiology Institute, Duke University, Durham, NC, United States) for providing us with the INS-1 832/13 cells is acknowledged.

\section{SUPPLEMENTARY MATERIAL}

The Supplementary Material for this article can be found online at: https://www.frontiersin.org/articles/10.3389/fcell.2021. 688885/full\#supplementary-material

Koepsell, H. (2020). Organic cation transporters in health and disease. Pharmacol. Rev. 72, 253-319. doi: 10.1124/pr.118.015578

Koepsell, H., Lips, K., and Volk, C. (2007). Polyspecific organic cation transporters: structure, function, physiological roles, and biopharmaceutical implications. Pharm. Res. 24, 1227-1251. doi: 10.1007/s11095-007-9254-z

Komdeur, R., Derksen, J., Legdeur, M. C., and Hylkema, B. S. (2007). Cisplatininduced hyperglycemic hyperosmolar coma. Neth. J. Med. 65, 36-37.

Konig, J., Zolk, O., Singer, K., Hoffmann, C., and Fromm, M. F. (2011). Double-transfected MDCK cells expressing human OCT1/MATE1 or OCT2/MATE1: determinants of uptake and transcellular translocation of organic cations. Br. J. Pharmacol. 163, 546-555. doi: 10.1111/j.1476-5381.2010. 01052.x

Leturque, A., Brot-Laroche, E., and Le, G. M. (2009). GLUT2 mutations, translocation, and receptor function in diet sugar managing. Am. J. Physiol. Endocrinol. Metab. 296, E985-E992.

Marks, J., Carvou, N. J., Debnam, E. S., Srai, S. K., and Unwin, R. J. (2003). Diabetes increases facilitative glucose uptake and GLUT2 expression at the rat proximal tubule brush border membrane. J. Physiol. 553(Pt 1), 137-145. doi: 10.1113/jphysiol.2003.046268

Motohashi, H., and Inui, K. (2016). "Pharmacological and toxicological significance of the organic cation transporters OCT and MATE: Drug disposition, interaction and toxicity," in Organic Cation Transporters, eds G. Ciarimboli, S. Gautron, and E. Schlatter (Cham: Springer), 73-92. doi: 10.1007/ 978-3-319-23793-0_3

Olson, L. K., Qian, J., and Poitout, V. (1998). Glucose rapidly and reversibly decreases INS-1 cell insulin gene transcription via decrements in STF-1 and C1 activator transcription factor activity. Mol. Endocrinol. 12, 207-219. doi: 10.1210/mend.12.2.0066

Pernicova, I., and Korbonits, M. (2014). Metformin-mode of action and clinical implications for diabetes and cancer. Nat. Rev. Endocrinol. 10, 143-156. doi: 10.1038/nrendo.2013.256

Snieder, B., Brast, S., Grabner, A., Buchholz, S., Schroter, R., Spoden, G. A., et al. (2019). Identification of the Tetraspanin CD9 as an interaction partner of organic cation transporters 1 and 2. SLAS Discov. 24, 904-914. doi: 10.1177/ 2472555219859837 
Song, I. S., Shin, H. J., and Shin, J. G. (2008). Genetic variants of organic cation transporter 2 (OCT2) significantly reduce metformin uptake in oocytes. Xenobiotica 38, 1252-1262. doi: 10.1080/004982508021 30039

Sprowl, J. A., Ciarimboli, G., Lancaster, C. S., Giovinazzo, H., Gibson, A. A., Du, G., et al. (2013). Oxaliplatin-induced neurotoxicity is dependent on the organic cation transporter OCT2. Proc. Natl. Acad. Sci. U.S.A. 110, 11199-11204. doi: $10.1073 /$ pnas.1305321110

Thorens, B. (2015). GLUT2, glucose sensing and glucose homeostasis. Diabetologia 58, 221-232. doi: 10.1007/s00125-014-3451-1

Wilde, S., Schlatter, E., Koepsell, H., Edemir, B., Reuter, S., Pavenstädt, H., et al. (2009). Calmodulin-associated post-translational regulation of rat organic cation transporter 2 in the kidney is gender dependent. Cell Mol. Life Sci. 66, 1729-1740. doi: 10.1007/s00018-009-9145-Z

Wittwer, M. B., Zur, A. A., Khuri, N., Kido, Y., Kosaka, A., Zhang, X., et al. (2013). Discovery of potent, selective multidrug and toxin extrusion transporter 1 (MATE1, SLC47A1) inhibitors through prescription drug profiling and computational modeling. J. Med. Chem. 56, 781-795. doi: 10.1021/jm30 $1302 \mathrm{~s}$

Wu, C., Qiu, S., Zhu, X., Lin, H., and Li, L. (2018). OCT1-mediated metformin uptake regulates pancreatic stellate cell activity. Cell Physiol. Biochem. 47, 1711-1720. doi: 10.1159/000491003

Yadav, Y. C. (2019). Effect of cisplatin on pancreas and testies in Wistar rats: biochemical parameters and histology. Heliyon 5:e02247. doi: 10.1016/j. heliyon.2019.e02247
Yang, X., Xu, Z., Zhang, C., Cai, Z., and Zhang, J. (2017). Metformin, beyond an insulin sensitizer, targeting heart and pancreatic beta cells. Biochim. Biophys. Acta Mol. Basis Dis. 1863, 1984-1990. doi: 10.1016/j.bbadis.2016.09.019

Zazuli, Z., Duin, N. J. C. B., Jansen, K., Vijverberg, S. J. H., Maitland-van der Zee, A. H., and Masereeuw, R. (2020). The impact of genetic polymorphisms in organic cation transporters on renal drug disposition. Int. J. Mol. Sci. 21:6627. doi: $10.3390 /$ ijms 21186627

Zhang, L., Gorset, W., Dresser, M. J., and Giacomini, K. M. (1999). The interaction of $\mathrm{n}$-tetraalkylammonium compounds with a human organic cation transporter, hOCT1. J. Pharmacol. Exp. Ther. 288, 1192-1198.

Zhou, K., Yee, S. W., Seiser, E. L., van, L. N., Tavendale, R., Bennett, A. J., et al. (2016). Variation in the glucose transporter gene SLC2A2 is associated with glycemic response to metformin. Nat. Genet. 48, 1055-1059.

Conflict of Interest: The authors declare that the research was conducted in the absence of any commercial or financial relationships that could be construed as a potential conflict of interest.

Copyright (c) 2021 Schorn, Dicke, Neugebauer, Schröter, Friedrich, Reuter and Ciarimboli. This is an open-access article distributed under the terms of the Creative Commons Attribution License (CC BY). The use, distribution or reproduction in other forums is permitted, provided the original author(s) and the copyright owner(s) are credited and that the original publication in this journal is cited, in accordance with accepted academic practice. No use, distribution or reproduction is permitted which does not comply with these terms. 